\title{
Fixed point theorems in ordered metric spaces via $w$-distances
}

\author{
Mohammad Imdad ${ }^{1 *}$ and Fayyaz Rouzkard ${ }^{1,2}$
}

\author{
"Correspondence: \\ mhimdad@yahoo.co.in \\ 'Department of Mathematics, \\ Aligarh Muslim University, Aligarh, \\ 202002, India \\ Full list of author information is \\ available at the end of the article
}

\begin{abstract}
The purpose of this paper is to prove some fixed point theorems in a complete metric space equipped with a partial ordering employing generalized distances together with altering distance functions.
\end{abstract}

MSC: $54 \mathrm{H} 25 ; 47 \mathrm{H} 10$

Keywords: ordered set; fixed point; complete metric space; altering functions; $w$-distance; nondecreasing map; orbitally continuous; orbitally $\mathcal{U}$-continuous

\section{Introduction}

The existing literature of metric fixed point theory contains numerous noted generalizations of the Banach contraction mapping principle (e.g., [1] and [2]). One variety of such generalizations is the contractive fixed point theorems contained in Khan et al. [1] wherein the authors utilized altering functions to alter the distance between two points in a metric space. Such altering functions are also sometimes referred to as control functions.

The following altering distance function is instrumental in our forthcoming results.

Definition A $(c f .[1])$ A map $\phi:[0, \infty) \rightarrow[0, \infty)$ is said to be an altering distance function if

(a) $\phi$ is continuous and nondecreasing and

(b) $\phi(t)=0$ if and only if $t=0$.

Using the function $\phi$, Khan et al. [1] proved the following result.

Theorem A $(c f .[1])$ Let $\mathcal{T}$ be a self-mapping defined on a complete metric space $(\mathcal{X}, d)$ satisfying the condition

$$
\phi(d(\mathcal{T} x, \mathcal{T} y)) \leq c \cdot \phi(d(x, y))
$$

for $x, y \in \mathcal{X}$ and $0<c<1$, where $\phi$ is the earlier described altering distance function. Then $\mathcal{T}$ has a unique fixed point.

In the recent past, the idea of altering function has been utilized by many researchers (e.g., [1-12]). Quite recently, Alber and Guerre-Delabriere [13] initiated the study of weakly contractive mappings which were initially confined to Hilbert spaces. Rhoades [11] utilized this idea in the context of complete metric spaces and proved the following interesting theorem.

\section{Springer}

(C) 2012 Imdad and Rouzkard; licensee Springer. This is an Open Access article distributed under the terms of the Creative Commons Attribution License (http://creativecommons.org/licenses/by/2.0), which permits unrestricted use, distribution, and reproduction in any medium, provided the original work is properly cited. 
Theorem B (cf. [11]) Let $\mathcal{T}$ be a self-mapping defined on a complete metric space $(\mathcal{X}, d)$ satisfying the condition

$$
d(\mathcal{T} x, \mathcal{T} y) \leq d(x, y)-\phi(d(x, y))
$$

for $x, y \in \mathcal{X}$, where $\phi$ is the earlier described altering distance function. Then $\mathcal{T}$ has a unique fixed point.

In fact, Alber and Guerre-Delabriere assumed the additional assumption $\lim _{t \rightarrow \infty} \phi(t)=$ $\infty$ (on $\phi)$. But Rhoades [11] proved his theorem without this requirement on $\phi$.

In [5], Dutta and Choudhury presented a generalization of Theorem B by proving the following result.

Theorem $\mathbf{C}(c f$. [5]) Let $\mathcal{T}$ be a self-mapping defined on a complete metric space $(\mathcal{X}, d)$ satisfying the condition

$$
\psi(d(\mathcal{T} x, \mathcal{T} y)) \leq \psi(d(x, y))-\phi(d(x, y)), \quad \text { for } x, y \in \mathcal{X},
$$

where $\psi$ and $\phi$ are altering distance functions. Then $\mathcal{T}$ has a unique fixed point.

The purpose of this paper is to prove some fixed point theorems in ordered metric spaces employing a $w$-distance as well as altering functions. Recall that the concept of $w$-distance was initiated by Kada, Suzuki, and Takahashi [7] and was primarily utilized to improve Caristi's fixed point theorem [4], Ekeland's variational principle [6], and the nonconvex minimization theorems whose descriptions and details are available in Takahashi [14]. The existence of a fixed point on partially ordered metric spaces has been a relatively new development in metric fixed point theory. In [10], Ran and Reurings proved an analogue of Banach's fixed point theorem in a partially ordered metric space besides discussing some applications to matrix equations. In fact, Ran and Reurings have weakened the usual contraction condition but merely up to monotone operators. Proving new fixed point theorems in an ordered metric space setting to improve earlier stated theorems have been a subject of vigorous research interest; for the literature of this kind one can be referred to $[5,8,9,15]$. Our results, in this paper, not only generalize the analogous fixed point theorems but are relatively simpler and more natural than the related ones. Our improvements in this paper are indeed four-fold:

(i) a generalized distance is used instead of metric,

(ii) a relatively more general contraction condition is used,

(iii) the continuity of the involved mapping is weakened to orbital continuity, and

(iv) the comparability conditions used by earlier authors are also sharpened.

\section{Preliminaries}

Before presenting our results, we collect relevant definitions and results which will be needed in the proof of our main results.

Definition 1 Let $\mathcal{X}$ be a nonempty set. Then $(\mathcal{X}, d, \preceq)$ is called a partially ordered metric space if

(i) $(\mathcal{X}, \preceq)$ is a partially ordered set and

(ii) $(\mathcal{X}, d)$ is a metric space. 
Definition 2 Let $(\mathcal{X}, \preceq)$ be a partially ordered set. Then

(a) elements $x, y \in \mathcal{X}$ are called comparable with respect to ' $\preceq$ ' if either $x \preceq y$ or $y \preceq x$;

(b) a mapping $\mathcal{T}: \mathcal{X} \rightarrow \mathcal{X}$ is called nondecreasing with respect to ' $\preceq$ ' if $x \preceq y$ implies $\mathcal{T} x \preceq \mathcal{T} y$.

Definition $3[7,16]$ Let $(\mathcal{X}, d)$ be a metric space. Then a function $p: \mathcal{X} \times \mathcal{X} \rightarrow[0, \infty)$ is called a $w$-distance on $\mathcal{X}$ if the following conditions are satisfied:

(a) $p(x, z) \leq p(x, y)+p(y, z)$ for any $x, y, z \in \mathcal{X}$,

(b) for any $x \in \mathcal{X}, p(x, \cdot): \mathcal{X} \rightarrow[0, \infty)$ is lower semi-continuous (i.e., if $x \in \mathcal{X}$ and $y_{n} \rightarrow y$ in $\mathcal{X}$, then $\left.p(x, y) \leq \liminf _{n} p\left(x, y_{n}\right)\right)$,

(c) for any $\epsilon>0$, there exists $\delta>0$ such that $p(x, z) \leq \delta$ and $p(z, y) \leq \delta$ imply $d(x, y) \leq \epsilon$.

Clearly, every metric is a $w$-distance but not conversely. The following example substantiates this fact.

Example 1 Let $(\mathcal{X}, d)$ be a metric space. A function $p: \mathcal{X} \times \mathcal{X} \rightarrow[0, \infty)$ defined by $p(x, y)=k$ for every $x, y \in \mathcal{X}$ is a $w$-distance on $\mathcal{X}$, where $k$ is a positive real number. But $p$ is not a metric since $p(x, x)=k \neq 0$ for any $x \in \mathcal{X}$.

Definition 4 Let $\mathcal{T}: \mathcal{X} \rightarrow \mathcal{X}$ be a function. Then

(a) $\mathcal{F}_{\mathcal{T}}=\{x \in \mathcal{X} \mid x=\mathcal{T}(x)\}$ (i.e., $\mathcal{F}_{\mathcal{T}}$ is the set of fixed points of $\mathcal{T}$ ),

(b) the function $\mathcal{T}$ is called a Picard operator (briefly, PO) if there exists $x^{*} \in \mathcal{X}$ such that $\mathcal{F}_{\mathcal{T}}=\left\{x^{*}\right\}$ and $\left\{\mathcal{T}^{n}(x)\right\}$ converges to $x^{*}$ for all $x \in \mathcal{X}$,

(c) the function $\mathcal{T}$ is called orbitally $\mathcal{U}$-continuous for any $\mathcal{U} \subset \mathcal{X} \times \mathcal{X}$ if for any $x \in \mathcal{X}$, $\mathcal{T}^{n_{i}}(x) \rightarrow a \in \mathcal{X}$ as $i \rightarrow \infty$ and $\left(\mathcal{T}^{n_{i}}(x), a\right) \in \mathcal{U}$ for any $i \in N$ imply that $\mathcal{T}^{n_{i}+1}(x) \rightarrow \mathcal{T} a$ as $i \rightarrow \infty$.

Let $(\mathcal{X}, \preceq)$ be a partially ordered set. Let us denote by $\mathcal{X}_{\leq}$the subset of $\mathcal{X} \times \mathcal{X}$ defined by

$$
\mathcal{X}_{\leq}=\{(x, y) \in \mathcal{X} \times \mathcal{X} \mid x \preceq y \text { or } y \preceq x\} .
$$

Definition 5 A map $\mathcal{T}: \mathcal{X} \rightarrow \mathcal{X}$ is said to be orbitally continuous if $x \in \mathcal{X}$ and $\mathcal{T}^{n_{i}}(x) \rightarrow$ $a \in \mathcal{X}$ as $i \rightarrow \infty$ imply that $\mathcal{T}^{n_{i}+1}(x) \rightarrow \mathcal{T} a$ as $i \rightarrow \infty$.

The following two lemmas are crucial in the proofs of our main results.

Lemma $1[7,17]$ Let $(\mathcal{X}, d)$ be a metric space equipped with a w-distance $p$. Let $\left\{x_{n}\right\}$ and $\left\{y_{n}\right\}$ be sequences in $\mathcal{X}$, whereas $\left\{\alpha_{n}\right\}$ and $\left\{\beta_{n}\right\}$ be sequences in $[0, \infty)$ converging to zero. Then the following conditions hold (for $x, y, z \in \mathcal{X}$ ):

(i) if $p\left(x_{n}, y\right) \leq \alpha_{n}$ and $p\left(x_{n}, z\right) \leq \beta_{n}$ for $n \in N$, then $y=z$. In particular, if $p(x, y)=0$ and $p(x, z)=0$, then $y=z$,

(ii) if $p\left(x_{n}, y_{n}\right) \leq \alpha_{n}$ and $p\left(x_{n}, z\right) \leq \beta_{n}$ for $n \in N$, then $\lim _{n \rightarrow \infty} d\left(y_{n}, z\right)=0$,

(iii) if $p\left(x_{n}, x_{m}\right) \leq \alpha_{n}$ for $n, m \in N$ with $m>n$, then $\left\{x_{n}\right\}$ is a Cauchy sequence,

(iv) if $p\left(y, x_{n}\right) \leq \alpha_{n}$ for $n \in N$, then $\left\{x_{n}\right\}$ is a Cauchy sequence. 
Lemma 2 [7] Let $p$ be a $w$-distance on a metric space $(\mathcal{X}, d)$ and $\left\{x_{n}\right\}$ be a sequence in $\mathcal{X}$ such that for each $\epsilon>0$, there exists $N_{\epsilon} \in N$ such that $m>n>N_{\epsilon}$ implies $p\left(x_{n}, x_{m}\right)<\epsilon$ (or $\left.\lim _{m, n} p\left(x_{n}, x_{m}\right)=0\right)$. Then $\left\{x_{n}\right\}$ is a Cauchy sequence.

\section{Main results}

Now, we present our main result as follows.

Theorem 1 Let $(\mathcal{X}, d, \preceq)$ be a complete partially ordered metric space equipped with a $w$-distance $p$ and $\mathcal{T}: \mathcal{X} \rightarrow \mathcal{X}$ be a nondecreasing mapping. Suppose that

(a) there exists $x_{0} \in \mathcal{X}$ such that $\left(x_{0}, \mathcal{T} x_{0}\right) \in \mathcal{X}_{\leq}$,

(b) there exist two altering distance functions $\psi$, $\phi$ such that

$$
\psi(p(\mathcal{T} x, \mathcal{T} y)) \leq \psi\left(\mathcal{M}_{x, y}\right)-\phi\left(\mathcal{M}_{x, y}\right)
$$

for all $(x, y) \in \mathcal{X}_{\leq}$, where

$$
\mathcal{M}_{x, y}=\max \{p(x, y), \min \{p(x, \mathcal{T} x), p(y, \mathcal{T} y), p(\mathcal{T} x, x), p(\mathcal{T} y, y)\}\}
$$

(c) either $\mathcal{T}$ is orbitally continuous at $x_{0}$ or

$\left(c^{\prime}\right) \mathcal{T}$ is orbitally $\mathcal{X}_{\leq}$-continuous, and there exists a subsequence $\left\{\mathcal{T}^{n_{k}} x_{0}\right\}$ of $\left\{\mathcal{T}^{n} x_{0}\right\}$ converging to $x^{*}$ such that $\left(\mathcal{T}^{n_{k}} x_{0}, x^{*}\right) \in \mathcal{X}_{\leq}$for any $k \in N$.

Then $\mathcal{F}_{\mathcal{T}} \neq \emptyset$.

Proof If $x_{0}=\mathcal{T} x_{0}$ for some $x_{0} \in \mathcal{X}$, then there is nothing to prove. Otherwise, let there be $x_{0} \in \mathcal{X}$ such that $x_{0} \neq \mathcal{T} x_{0}$ and $\left(x_{0}, \mathcal{T} x_{0}\right) \in \mathcal{X}_{\leq}$. Owing to monotonicity of $\mathcal{T}$, we can write $\left(\mathcal{T} x_{0}, \mathcal{T}^{2} x_{0}\right) \in \mathcal{X}_{\leq}$. Continuing this process inductively, we obtain

$$
\left(\mathcal{T}^{n} x_{0}, \mathcal{T}^{m} x_{0}\right) \in \mathcal{X}_{\leq}
$$

for any $n, m \in N$. Now, we proceed to show that

$$
\lim _{n \rightarrow \infty} p\left(\mathcal{T}^{n} x_{0}, \mathcal{T}^{n+1} x_{0}\right)=0
$$

Write $p_{0}=p\left(x_{0}, \mathcal{T} x_{0}\right)$ and $p_{n}=p\left(\mathcal{T}^{n} x_{0}, \mathcal{T}^{n+1} x_{0}\right)$ for any $n \in N$.

By using condition (b), we have

$$
\begin{aligned}
\psi\left(p_{n}\right)= & \psi\left(p\left(\mathcal{T}^{n} x_{0}, \mathcal{T}^{n+1} x_{0}\right)\right) \\
\leq & \psi\left(\operatorname { m a x } \left\{p\left(\mathcal{T}^{n-1} x_{0}, \mathcal{T}^{n} x_{0}\right), \min \left\{p\left(\mathcal{T}^{n-1} x_{0}, \mathcal{T}^{n} x_{0}\right),\right.\right.\right. \\
& \left.\left.\left.p\left(\mathcal{T}^{n} x_{0}, \mathcal{T}^{n+1} x_{0}\right), p\left(\mathcal{T}^{n} x_{0}, \mathcal{T}^{n-1} x_{0}\right), p\left(\mathcal{T}^{n+1} x_{0}, \mathcal{T}^{n} x_{0}\right)\right\}\right\}\right) \\
& -\phi\left(\operatorname { m a x } \left\{p\left(\mathcal{T}^{n-1} x_{0}, \mathcal{T}^{n} x_{0}\right), \min \left\{p\left(\mathcal{T}^{n-1} x_{0}, \mathcal{T}^{n} x_{0}\right),\right.\right.\right. \\
& \left.\left.\left.p\left(\mathcal{T}^{n} x_{0}, \mathcal{T}^{n+1} x_{0}\right), p\left(\mathcal{T}^{n} x_{0}, \mathcal{T}^{n-1} x_{0}\right), p\left(\mathcal{T}^{n+1} x_{0}, \mathcal{T}^{n} x_{0}\right)\right\}\right\}\right)
\end{aligned}
$$

so that

$$
\psi\left(p_{n}\right) \leq \psi\left(p_{n-1}\right)-\phi\left(p_{n-1}\right)
$$

for any $n \in \mathbb{N}$. 
Also,

$$
\psi\left(p_{n}\right) \leq \psi\left(p_{n-1}\right)-\phi\left(p_{n-1}\right) \leq \psi\left(p_{n-1}\right) .
$$

Therefore, $p_{n} \leq p_{n-1}$ for every $n \in \mathbb{N}$ (owing to monotonicity of $\psi$ ), i.e., the sequence $\left\{p_{n}\right\}$ is decreasing so that for the nonnegative decreasing sequence $\left\{p_{n}\right\}$, there exists some $r \geq 0$ such that

$$
\lim _{n \rightarrow \infty} p_{n}=\lim _{n \rightarrow \infty} p\left(\mathcal{T}^{n} x_{0}, \mathcal{T}^{n+1} x_{0}\right)=r
$$

Assume that $r>0$. On letting $n \rightarrow \infty$ in (3.2) besides using (3.3), we get

$$
\psi(r) \leq \psi(r)-\phi(r) \leq \psi(r),
$$

which amounts to say that $\phi(r)=0$. As $\phi$ is an altering (distance) function, $r=0$, which is a contradiction to nonzeroness of $r$ yielding thereby

$$
\lim _{n \rightarrow \infty} p_{n}=\lim _{n \rightarrow \infty} p\left(\mathcal{T}^{n} x_{0}, \mathcal{T}^{n+1} x_{0}\right)=0,
$$

which establishes (3.1).

Proceeding with earlier lines, we can also show that

$$
\lim _{n \rightarrow \infty} p\left(\mathcal{T}^{n+1} x_{0}, \mathcal{T}^{n} x_{0}\right)=0
$$

Write $p_{0}=p\left(\mathcal{T} x_{0}, x_{0}\right)$ and $p_{n}=p\left(\mathcal{T}^{n+1} x_{0}, \mathcal{T}^{n} x_{0}\right)$ for any $n \in N$.

Now, using (b), we get

$$
\begin{aligned}
\psi\left(p_{n}\right)= & \psi\left(p\left(\mathcal{T}^{n+1} x_{0}, \mathcal{T}^{n} x_{0}\right)\right) \\
\leq & \psi\left(\operatorname { m a x } \left\{p\left(\mathcal{T}^{n} x_{0}, \mathcal{T}^{n-1} x_{0}\right), \min \left\{p\left(\mathcal{T}^{n} x_{0}, \mathcal{T}^{n+1} x_{0}\right),\right.\right.\right. \\
& \left.\left.\left.p\left(\mathcal{T}^{n-1} x_{0}, \mathcal{T}^{n} x_{0}\right), p\left(\mathcal{T}^{n+1} x_{0}, \mathcal{T}^{n} x_{0}\right), p\left(\mathcal{T}^{n} x_{0}, \mathcal{T}^{n-1} x_{0}\right)\right\}\right\}\right) \\
& -\phi\left(\operatorname { m a x } \left\{p\left(\mathcal{T}^{n} x_{0}, \mathcal{T}^{n-1} x_{0}\right), \min \left\{p\left(\mathcal{T}^{n} x_{0}, \mathcal{T}^{n+1} x_{0}\right),\right.\right.\right. \\
& \left.\left.\left.p\left(\mathcal{T}^{n-1} x_{0}, \mathcal{T}^{n} x_{0}\right), p\left(\mathcal{T}^{n+1} x_{0}, \mathcal{T}^{n} x_{0}\right), p\left(\mathcal{T}^{n} x_{0}, \mathcal{T}^{n-1} x_{0}\right)\right\}\right\}\right) \\
= & \psi\left(p_{n-1}\right)-\phi\left(p_{n-1}\right),
\end{aligned}
$$

so that

$$
\psi\left(p_{n}\right) \leq \psi\left(p_{n-1}\right)-\phi\left(p_{n-1}\right) \leq \psi\left(p_{n-1}\right),
$$

which amounts to say that $p_{n} \leq p_{n-1}$, i.e., a nonnegative sequence $\left\{p_{n}\right\}$ is decreasing. As earlier, we have

$$
\lim _{n \rightarrow \infty} p\left(\mathcal{T}^{n+1} x_{0}, \mathcal{T}^{n} x_{0}\right)=0
$$


which proves (3.4). Now, we proceed to show

$$
\lim _{n, m \rightarrow \infty} p\left(\mathcal{T}^{n} x_{0}, \mathcal{T}^{m} x_{0}\right)=0
$$

Suppose (3.5) is untrue. Then we can find a $\delta>0$ with sequences $\left\{m_{k}\right\}_{k=1}^{\infty},\left\{n_{k}\right\}_{k=1}^{\infty}$ such that

$$
p\left(\mathcal{T}^{n_{k}} x_{0}, \mathcal{T}^{m_{k}} x_{0}\right) \geq \delta, \quad \text { for all } k \in\{1,2,3, \ldots\}
$$

wherein $m_{k}>n_{k}$. By (3.1) there exists $k_{0} \in \mathbb{N}$ such that $n_{k}>k_{0}$ implies

$$
p\left(\mathcal{T}^{n_{k}} x_{0}, \mathcal{T}^{n_{k}+1} x_{0}\right)<\delta
$$

Notice that in view of (3.6) and (3.7), $m_{k} \neq n_{k+1}$. We can assume that $m_{k}$ is a minimum index such that (3.6) holds so that

$$
p\left(\mathcal{T}^{n_{k}} x_{0}, \mathcal{T}^{r} x_{0}\right)<\delta, \quad \text { for } r \in\left\{n_{k+1}, n_{k+2}, \ldots, m_{k}-1\right\} \text {, }
$$

which in view of (3.6) gives rise to

$$
\begin{aligned}
o & <\delta \leq p\left(\mathcal{T}^{n_{k}} x_{0}, \mathcal{T}^{m_{k}} x_{0}\right) \\
& \leq p\left(\mathcal{T}^{n_{k}} x_{0}, \mathcal{T}^{m_{k}-1} x_{0}\right)+p\left(\mathcal{T}^{m_{k}-1} x_{0}, \mathcal{T}^{m_{k}} x_{0}\right) \\
& <\delta+p\left(\mathcal{T}^{m_{k}-1} x_{0}, \mathcal{T}^{m_{k}} x_{0}\right)
\end{aligned}
$$

so that

$$
\lim _{k \rightarrow \infty} p\left(\mathcal{T}^{n_{k}} x_{0}, \mathcal{T}^{m_{k}} x_{0}\right)=\delta
$$

Next, we show that

$$
\limsup p\left(\mathcal{T}^{n_{k}+1} x_{0}, \mathcal{T}^{m_{k}+1} x_{0}\right)=\epsilon<\delta
$$

If $\lim \sup _{k} p\left(\mathcal{T}^{n_{k}+1} x_{0}, \mathcal{T}^{m_{k}+1} x_{0}\right)=\epsilon \geq \delta$, then there exists $\left\{k_{r}\right\}_{r=1}^{\infty}$ such that

$$
\lim _{r \rightarrow \infty} p\left(\mathcal{T}^{n_{k_{r}+1}} x_{0}, \mathcal{T}^{m_{k_{r}}+1} x_{0}\right)=\epsilon \geq \delta
$$

Since $\psi$ is continuous and nondecreasing and also $\left(\mathcal{T}^{n_{k_{r}}} x_{0}, \mathcal{T}^{m_{k_{r}}} x_{0}\right) \in \mathcal{X}_{\leq}$, on using condition (b), one gets

$$
\psi\left(p\left(\mathcal{T}^{n_{k_{r}}+1} x_{0}, \mathcal{T}^{m_{k_{r}}+1} x_{0}\right)\right) \leq \psi\left(\mathcal{M}_{\mathcal{T}^{n_{k_{r} x_{0}}}, \mathcal{T}^{m_{k_{r} x_{0}}}}\right)-\phi\left(\mathcal{M}_{\mathcal{T}^{n_{k_{r} x_{0}}}, \mathcal{T}^{m_{k_{r} x_{0}}}}\right)
$$

with

$$
\begin{aligned}
\mathcal{M}_{\mathcal{T}^{k_{k x_{0}},}} \mathcal{T}^{m_{k_{r} x_{0}}}= & \max \left\{p\left(\mathcal{T}^{n_{k_{r}} x_{0}}, \mathcal{T}^{m_{k_{r}}} x_{0}\right), \min \left\{p\left(\mathcal{T}^{n_{k_{r}}} x_{0}, \mathcal{T}^{n_{k_{r}}+1} x_{0}\right),\right.\right. \\
& \left.\left.p\left(\mathcal{T}^{m_{k_{r}}} x_{0}, \mathcal{T}^{m_{k_{r}}+1} x_{0}\right), p\left(\mathcal{T}^{n_{k_{r}}+1} x_{0}, \mathcal{T}^{n_{k_{r}}} x_{0}\right), p\left(\mathcal{T}^{m_{k_{r}}+1} x_{0}, \mathcal{T}^{m_{k_{r}}} x_{0}\right)\right\}\right\},
\end{aligned}
$$


implying thereby

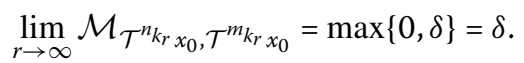

Letting $k \rightarrow \infty$ in (3.8) and using (3.9), we get

$$
\psi(\delta) \leq \psi(\epsilon) \leq \psi(\delta)-\phi(\delta) \leq \psi(\delta)
$$

so that $\phi(\delta)=0$ implying thereby $\delta=0$, which is a contradiction. Hence,

$$
\limsup _{k} p\left(\mathcal{T}^{n_{k}+1} x_{0}, \mathcal{T}^{m_{k}+1} x_{0}\right)<\delta
$$

and we have

$$
\begin{aligned}
o & <\delta \leq p\left(\mathcal{T}^{n_{k}} x_{0}, \mathcal{T}^{m_{k}} x_{0}\right) \\
& \leq p\left(\mathcal{T}^{n_{k}} x_{0}, \mathcal{T}^{n_{k}+1} x_{0}\right)+p\left(\mathcal{T}^{n_{k}+1} x_{0}, \mathcal{T}^{m_{k}+1} x_{0}\right)+p\left(\mathcal{T}^{m_{k}+1} x_{0}, \mathcal{T}^{m_{k}} x_{0}\right) .
\end{aligned}
$$

Therefore, owing to (3.1) and (3.4), we have

$$
\begin{aligned}
o & <\delta \\
& \leq \lim _{k \rightarrow \infty} p\left(\mathcal{T}^{n_{k}} x_{0}, \mathcal{T}^{n_{k}+1} x_{0}\right)+\limsup _{k \rightarrow \infty} p\left(\mathcal{T}^{n_{k}+1} x_{0}, \mathcal{T}^{m_{k}+1} x_{0}\right)+\lim _{k \rightarrow \infty} p\left(\mathcal{T}^{m_{k}+1} x_{0}, \mathcal{T}^{m_{k}} x_{0}\right) \\
& =\limsup _{k \rightarrow \infty} p\left(\mathcal{T}^{n_{k}+1} x_{0}, \mathcal{T}^{m_{k}+1} x_{0}\right)<\delta,
\end{aligned}
$$

which is a contradiction. Hence, (3.5) holds. Owing to Lemma $2,\left\{\mathcal{T}^{n} x_{0}\right\}$ is a Cauchy sequence in $\mathcal{X}$. Since $\mathcal{X}$ is a complete metric space, there exists $x^{*}$ such that $\lim _{n \rightarrow \infty} \mathcal{T}^{n} x_{0}=x^{*}$.

Now, we show that $x^{\prime \prime}$ is a fixed point of $\mathcal{T}$. If (c) holds, then $\mathcal{T}^{n+1} x_{0} \rightarrow \mathcal{T} x^{\prime \prime}$ (as $n \rightarrow \infty$ ). By lower semi-continuity of $p\left(\mathcal{T}^{n} x_{0}, \cdot\right)$, we have

$$
\begin{aligned}
& p\left(\mathcal{T}^{n} x_{0}, x^{*}\right) \leq \liminf _{m \rightarrow \infty} p\left(\mathcal{T}^{n} x_{0}, \mathcal{T}^{m} x_{0}\right)=\alpha_{n} \quad \text { (say), } \\
& p\left(\mathcal{T}^{n} x_{0}, \mathcal{T} x^{*}\right) \leq \liminf _{m \rightarrow \infty} p\left(\mathcal{T}^{n} x_{0}, \mathcal{T}^{m+1} x_{0}\right)=\beta_{n} \quad \text { (say). }
\end{aligned}
$$

On using (3.5), we have $\lim _{n \rightarrow \infty} \alpha_{n}=\lim _{n \rightarrow \infty} \beta_{n}=0$. Now, in view of Lemma 1, we conclude that

$$
\mathcal{T} x^{*}=x^{*}
$$

Next, suppose that $\left(\mathrm{c}^{\prime}\right)$ holds. Since $\left\{\mathcal{T}^{n_{k}} x_{0}\right\}$ converges to $x^{*},\left(\mathcal{T}^{n_{k}} x_{0}, x^{*}\right) \in \mathcal{X}_{\leq}$and $\mathcal{T}$ is $\mathcal{X}_{\leq}-$ continuous, it follows that $\left\{\mathcal{T}^{n_{k}+1} x_{0}\right\}$ converges to $\mathcal{T} x^{*}$. As earlier, by lower semi-continuity of $p\left(\mathcal{T}^{n} x_{0}, \cdot\right)$, we conclude that $\mathcal{T} x^{n}=x^{n}$. This completes the proof.

Setting $\psi=\mathcal{I}$ (the identity mapping) in Theorem 1, we deduce the following corollary.

Corollary 1 Let $(\mathcal{X}, d, \preceq)$ be a complete partially ordered metric space equipped with a $w$-distance $p$ and $\mathcal{T}: \mathcal{X} \rightarrow \mathcal{X}$ be a nondecreasing mapping. Suppose that 
(a) there exists $x_{0} \in \mathcal{X}$ such that $\left(x_{0}, \mathcal{T} x_{0}\right) \in \mathcal{X}_{\leq}$,

(b) there exists an altering distance function $\phi$ such that

$$
p(\mathcal{T} x, \mathcal{T} y) \leq \mathcal{M}_{x, y}-\phi\left(\mathcal{M}_{x, y}\right)
$$

for all $(x, y) \in \mathcal{X}_{\leq}$, where

$$
\mathcal{M}_{x, y}=\max \{p(x, y), \min \{p(x, \mathcal{T} x), p(y, \mathcal{T} y), p(\mathcal{T} x, x), p(\mathcal{T} y, y)\}\}
$$

(c) either $\mathcal{T}$ is orbitally continuous at $x_{0}$ or

$\left(\mathrm{c}^{\prime}\right) \mathcal{T}$ is orbitally $\mathcal{X}_{\leq}$-continuous, and there exists a subsequence $\left\{\mathcal{T}^{n_{k}} x_{0}\right\}$ of $\left\{\mathcal{T}^{n} x_{0}\right\}$ which converges to $x^{*}$ such that $\left(\mathcal{T}^{n_{k}} x_{0}, x^{*}\right) \in \mathcal{X}_{\leq}$for any $k \in N$.

Then $\mathcal{F}_{\mathcal{T}} \neq \emptyset$.

Choosing $\psi=\mathcal{I}$ (the identity mapping) and $\phi(t)=(1-\alpha) t$ (for all $t \in[0, \infty))$ in Theorem 1 , we deduce the following corollary.

Corollary 2 Let $(\mathcal{X}, d, \preceq)$ be a complete partially ordered metric space equipped with a $w$-distance $p$ and $\mathcal{T}: \mathcal{X} \rightarrow \mathcal{X}$ be a nondecreasing mapping. Suppose that

(a) there exists $x_{0} \in \mathcal{X}$ such that $\left(x_{0}, \mathcal{T} x_{0}\right) \in \mathcal{X}_{\leq}$,

(b) iffor all $(x, y) \in \mathcal{X}_{\leq}$and $\alpha \in[0,1)$,

$$
p(\mathcal{T} x, \mathcal{T} y) \leq \alpha \mathcal{M}_{x, y}
$$

where

$$
\mathcal{M}_{x, y}=\max \{p(x, y), \min \{p(x, \mathcal{T} x), p(y, \mathcal{T} y), p(\mathcal{T} x, x), p(\mathcal{T} y, y)\}\}
$$

(c) either $\mathcal{T}$ is orbitally continuous at $x_{0}$ or

(c') $\mathcal{T}$ is orbitally $\mathcal{X}_{\leq}$-continuous, and there exists a subsequence $\left\{\mathcal{T}^{n_{k}} x_{0}\right\}$ of $\left\{\mathcal{T}^{n} x_{0}\right\}$ which converges to $x^{*}$ such that $\left(\mathcal{T}^{n_{k}} x_{0}, x^{*}\right) \in \mathcal{X}_{\leq}$for any $k \in N$.

Then $\mathcal{F}_{\mathcal{T}} \neq \emptyset$.

As an application of Corollary 2, we can also prove the following related result.

Theorem 2 Let $(\mathcal{X}, d, \preceq)$ be a complete partially ordered metric space equipped with a $w$-distance $p$ and $\mathcal{T}: \mathcal{X} \rightarrow \mathcal{X}$ be a nondecreasing mapping. Suppose that

(a) there exists $x_{0} \in \mathcal{X}$ such that $\left(x_{0}, \mathcal{T} x_{0}\right) \in \mathcal{X}_{\leq}$,

(b) for all $(x, y) \in \mathcal{X}_{\leq}$,

$$
p(\mathcal{T} x, \mathcal{T} y) \leq \alpha p(x, y)+\beta(\min \{p(x, \mathcal{T} x), p(y, \mathcal{T} y), p(\mathcal{T} x, x), p(\mathcal{T} y, y)\})
$$

where $\alpha, \beta \geq 0$ and $\alpha+\beta<1$,

(c) either $\mathcal{T}$ is orbitally continuous at $x_{0}$ or 
$\left(c^{\prime}\right) \mathcal{T}$ is orbitally $\mathcal{X}_{\leq}$-continuous, and there exists a subsequence $\left\{\mathcal{T}^{n_{k}} x_{0}\right\}$ of $\left\{\mathcal{T}^{n} x_{0}\right\}$ which converges to $x^{*}$ such that $\left(\mathcal{T}^{n_{k}} x_{0}, x^{*}\right) \in \mathcal{X}_{\leq}$for any $k \in N$.

Then $\mathcal{F}_{\mathcal{T}} \neq \emptyset$.

Proof On using condition (b), we can write

$$
\begin{aligned}
p(\mathcal{T} x, \mathcal{T} y) & \leq \alpha p(x, y)+\beta(\min \{p(x, \mathcal{T} x), p(y, \mathcal{T} y), p(\mathcal{T} x, x), p(\mathcal{T} y, y)\}) \\
& \leq(\alpha+\beta) \max \{p(x, y), \min \{p(x, \mathcal{T} x), p(y, \mathcal{T} y), p(\mathcal{T} x, x), p(\mathcal{T} y, y)\}\},
\end{aligned}
$$

where $k=\alpha+\beta \in[0,1)$. Therefore, all the conditions of Corollary 2 are satisfied, which ensures the conclusion.

Corollary 3 Let $(\mathcal{X}, d, \preceq)$ be a complete partially ordered metric space equipped with a $w$-distance $p$ and $\mathcal{T}: \mathcal{X} \rightarrow \mathcal{X}$ be a nondecreasing mapping. Suppose that

(a) there exists $x_{0} \in \mathcal{X}$ such that $\left(x_{0}, \mathcal{T} x_{0}\right) \in \mathcal{X}_{\leq}$,

(b) for all $(x, y) \in \mathcal{X}_{\leq}$,

$$
\int_{0}^{p(\mathcal{T} x, \mathcal{T} y)} \theta(\xi) d \xi \leq \alpha \int_{0}^{\max \{p(x, y), \min \{p(x, \mathcal{T} x), p(y, \mathcal{T} y), p(\mathcal{T} x, x), p(\mathcal{T} y, y)\}\}} \theta(\xi) d \xi,
$$

where $0 \leq \alpha<1$, and $\theta: \mathbb{R}^{+} \rightarrow \mathbb{R}^{+}$is a Lebesgue integrable mapping which is summable and $\int_{0}^{\epsilon} \theta(\xi) d \xi>0$ (for each $\left.\epsilon>0\right)$,

(c) either $\mathcal{T}$ is orbitally continuous at $x_{0}$ or

(c') $\mathcal{T}$ is orbitally $\mathcal{X}_{\leq}$-continuous, and there exists a subsequence $\left\{\mathcal{T}^{n_{k}} x_{0}\right\}$ of $\left\{\mathcal{T}^{n} x_{0}\right\}$ which converges to $x^{\prime \prime}$ such that $\left(\mathcal{T}^{n_{k}} x_{0}, x^{*}\right) \in \mathcal{X}_{\leq}$for any $k \in N$.

Then $\mathcal{F}_{\mathcal{T}} \neq \emptyset$.

Proof Choose $\psi(t)=\int_{0}^{t} \theta(\xi) d \xi$ and $\phi(t)=(1-\alpha) \int_{0}^{t} \theta(\xi) d \xi$ (for all $t \in[0, \infty)$ ). Clearly, $\psi$ and $\phi$ are altering distance functions. Now, in view of Theorem 1, result follows.

Remark 1 In Theorem 1, let $p=d$, and $\psi=\mathcal{I}$ (identity) and $\phi=(1-\alpha) t(0 \leq \alpha<1)$. Then Theorem 1 is the classical Banach fixed point theorem.

Lemma 3 Let $(\mathcal{X}, d, \preceq)$ be a complete partially ordered metric space and $\mathcal{T}: \mathcal{X} \rightarrow \mathcal{X}$ be a map wherein $p$ is a w-distance on $(\mathcal{X}, d)$. If

(a) $x^{*} \in \mathcal{F}_{\mathcal{T}}$,

(b) there exist two altering distance functions $\psi$, $\phi$ such that

$$
\psi(p(\mathcal{T} x, \mathcal{T} y)) \leq \psi\left(\mathcal{M}_{x, y}\right)-\phi\left(\mathcal{M}_{x, y}\right)
$$

for all $(x, y) \in \mathcal{X}_{\leq}$, with

$$
\mathcal{M}_{x, y}=\max \{p(x, y), \min \{p(x, \mathcal{T} x), p(y, \mathcal{T} y), p(\mathcal{T} x, x), p(\mathcal{T} y, y)\}\},
$$

then $p\left(x^{*}, x^{*}\right)=0$. 
Proof Suppose $p\left(x^{\prime \prime}, x^{\prime \prime}\right) \neq 0$. As $\left(x^{\prime \prime}, x^{*}\right) \in \mathcal{X}_{\leq}$and

$$
\begin{aligned}
\mathcal{M}_{x^{\prime \prime}, x^{*}} & =\max \left\{p\left(x^{*}, x^{*}\right), \min \left\{p\left(x^{*}, \mathcal{T} x^{*}\right), p\left(x^{*}, \mathcal{T} x^{*}\right), p\left(\mathcal{T} x^{*}, x^{*}\right), p\left(\mathcal{T} x^{*}, x^{*}\right)\right\}\right\} \\
& =p\left(x^{*}, x^{*}\right) .
\end{aligned}
$$

Therefore,

$$
\psi\left(p\left(\mathcal{T} x^{*}, \mathcal{T} x^{*}\right)\right)=\psi\left(p\left(x^{*}, x^{*}\right)\right) \leq \psi\left(p\left(x^{*}, x^{*}\right)\right)-\phi\left(p\left(x^{*}, x^{*}\right)\right) \leq \psi\left(p\left(x^{*}, x^{*}\right)\right),
$$

which amounts to say that $\phi\left(p\left(x^{*}, x^{*}\right)\right)=0$. As $\phi$ is an altering distance function, we infer that $p\left(x^{\prime \prime}, x^{\prime \prime}\right)=0$. This completes the proof.

In what follows, we give a sufficient condition for the uniqueness of a fixed point in Theorem 1 which runs as follows:

(A): for every $x, y \in \mathcal{X}$, there exists a lower bound or an upper bound.

In [9], it is proved that condition (A) is equivalent to the following one:

(B): for every $x, y \in \mathcal{X}$, there exists $z=c(x, y) \in \mathcal{X}$ for which $(x, z) \in \mathcal{X}_{\leq}$and $(y, z) \in \mathcal{X}_{\leq}$.

\section{Results with uniqueness}

Theorem 3 With the addition of condition (B) to the hypotheses of Theorem 1, the fixed point of $\mathcal{T}$ turns out to be unique. Moreover,

$$
\lim _{n \rightarrow \infty} \mathcal{T}^{n}(x)=x^{*}
$$

for every $x \in \mathcal{X}$ provided $x^{*} \in \mathcal{F}_{\mathcal{T}}$, i.e., the map $\mathcal{T}: \mathcal{X} \rightarrow \mathcal{X}$ is a Picard operator.

Proof Following the proof of Theorem $1, \mathcal{F}_{\mathcal{T}} \neq \emptyset$. Suppose there exist two fixed points $x^{*}$ and $y^{*}$ of $\mathcal{T}$ in $\mathcal{X}$. We distinguish two cases.

Case 1: If $\left(y^{*}, x^{*}\right) \in \mathcal{X}_{\leq}$, owing to condition (b) (of Theorem 1) and Lemma 3, we have

$$
\psi\left(p\left(\mathcal{T} y^{*}, \mathcal{T} x^{*}\right)\right) \leq \psi\left(\mathcal{M}_{y^{*}, x^{*}}\right)-\phi\left(\mathcal{M}_{y^{\prime \prime}, x^{*}}\right)
$$

As

$$
\begin{aligned}
\mathcal{M}_{y^{*}, x^{*}} & =\max \left\{p\left(y^{*}, x^{*}\right), \min \left\{p\left(y^{*}, \mathcal{T} y^{*}\right), p\left(x^{*}, \mathcal{T} x^{*}\right), p\left(\mathcal{T} y^{*}, y^{*}\right), p\left(\mathcal{T} x^{*}, x^{*}\right)\right\}\right\} \\
& =p\left(y^{*}, x^{*}\right),
\end{aligned}
$$

therefore

$$
\psi\left(p\left(\mathcal{T} y^{*}, \mathcal{T} x^{*}\right)\right) \leq \psi\left(p\left(y^{*}, x^{*}\right)\right)-\phi\left(p\left(y^{*}, x^{*}\right)\right) \leq \psi\left(p\left(y^{*}, x^{*}\right)\right)
$$

which amounts to say that $\phi\left(p\left(y^{*}, x^{*}\right)\right)=0$. As $\phi$ is an altering distance function, therefore, for every $n \in \mathbb{N}$,

$$
p\left(y^{*}, x^{*}\right)=0 \text {. }
$$


Also, in view of Lemma 3 , we get $p\left(y^{*}, y^{*}\right)=0$, and by using Lemma 1 , we have $y^{*}=x^{*}$, i.e., the fixed point of $\mathcal{T}$ is unique.

Case 2: If $\left(x^{*}, y^{*}\right) \notin \mathcal{X}_{\leq}$, then owing to condition (B), there exists $z \in \mathcal{X}$ such that $\left(x^{*}, z\right) \in$ $\mathcal{X}_{\leq}$and $\left(y^{\prime \prime}, z\right) \in \mathcal{X}_{\leq}$. As $\left(z, x^{\prime \prime}\right) \in \mathcal{X}_{\leq}$, due to monotonicity of $\mathcal{T}$, we get $\left(\mathcal{T}^{n-1} z, x^{*}\right) \in \mathcal{X}_{\leq}$for any $n \in \mathbb{N}$, and henceforth

$$
\psi\left(p\left(\mathcal{T}^{n} z, x^{*}\right)\right)=\psi\left(p\left(\mathcal{T}^{n} z, \mathcal{T} x^{*}\right)\right) \leq \psi\left(\mathcal{M}_{\mathcal{T}^{n-1} z, x^{*}}\right)-\phi\left(\mathcal{M}_{\mathcal{T}^{n-1} z, x^{*}}\right)
$$

with

$$
\begin{aligned}
& \mathcal{M}_{\mathcal{T}^{n-1} z, x^{*}} \\
& \quad=\max \left\{p\left(\mathcal{T}^{n-1} z, x^{*}\right), \min \left\{p\left(\mathcal{T}^{n-1} z, \mathcal{T}^{n} z\right), p\left(x^{*}, \mathcal{T} x^{*}\right), p\left(\mathcal{T}^{n} z, \mathcal{T}^{n-1} z\right), p\left(x^{*}, x^{*}\right)\right\}\right\} \\
& =p\left(\mathcal{T}^{n-1} z, x^{n}\right),
\end{aligned}
$$

therefore

$$
\begin{aligned}
\psi\left(p\left(\mathcal{T}^{n} z, x^{*}\right)\right) & =\psi\left(p\left(\mathcal{T}^{n} z, \mathcal{T} x^{*}\right)\right) \leq \psi\left(p\left(\mathcal{T}^{n-1} z, x^{*}\right)\right)-\phi\left(p\left(\mathcal{T}^{n-1} z, x^{*}\right)\right) \\
& \leq \psi\left(p\left(\mathcal{T}^{n-1} z, x^{*}\right)\right) .
\end{aligned}
$$

Since $\psi$ is a nondecreasing function, therefore $p\left(\mathcal{T}^{n} z, x^{n}\right) \leq p\left(\mathcal{T}^{n-1} z, x^{*}\right)$, i.e., the nonnegative sequence $\left\{p\left(\mathcal{T}^{n} z, x^{*}\right)\right\}$ is decreasing. As earlier, we have

$$
\lim _{n \rightarrow \infty} p\left(\mathcal{T}^{n} z, x^{*}\right)=0
$$

Also, since $\left(z, y^{*}\right) \in \mathcal{X}_{\leq}$, therefore proceeding as earlier, we can prove that

$$
\lim _{n \rightarrow \infty} p\left(\mathcal{T}^{n} z, y^{*}\right)=0
$$

By using this and Lemma 1 , we infer that $y^{*}=x^{*}$, i.e., the fixed point of $\mathcal{T}$ is unique.

Now, we proceed to show

$$
\lim _{n \rightarrow \infty} \mathcal{T}^{n}(x)=x^{*}
$$

for every $x \in \mathcal{X}$ provided $x^{*} \in \mathcal{F}_{\mathcal{T}}$. We distinguish two cases.

Case 1: Let $x \in \mathcal{X}$ and $\left(x^{*}, x\right) \in \mathcal{X}_{\leq}$. As earlier, we have

$$
\lim _{n \rightarrow \infty} p\left(\mathcal{T}^{n} x^{*}, \mathcal{T}^{n} x\right)=0
$$

Also, in view of Lemma 3, we have

$$
\lim _{n \rightarrow \infty} p\left(\mathcal{T}^{n} x^{*}, x^{*}\right)=0,
$$

and by using Lemma 1, we get

$$
\lim _{n \rightarrow \infty} \mathcal{T}^{n}(x)=x^{*} .
$$


Case 2: Let $x \in \mathcal{X}$ and $\left(x^{*}, x\right) \notin \mathcal{X}_{\leq}$. Owing to condition (B), there exists some $z$ in $\mathcal{X}$ such that $\left(x^{*}, z\right) \in \mathcal{X}_{\leq}$and $(x, z) \in \mathcal{X}_{\leq}$. As earlier, we can prove $\lim _{n \rightarrow \infty} p\left(\mathcal{T}^{n} z, x^{*}\right)=0$ and $\lim _{n \rightarrow \infty} p\left(x^{*}, \mathcal{T}^{n} z\right)=0$. By the triangular inequality,

$$
p\left(\mathcal{T}^{n} z, \mathcal{T}^{n} z\right) \leq p\left(\mathcal{T}^{n} z, x^{*}\right)+p\left(x^{*}, \mathcal{T}^{n} z\right)
$$

one gets

$$
\lim _{n \rightarrow \infty} p\left(\mathcal{T}^{n} z, \mathcal{T}^{n} z\right)=0
$$

Since $(x, z) \in \mathcal{X}_{\leq}$, due to monotonicity of $\mathcal{T}$, we can write $\left(\mathcal{T}_{x}, \mathcal{T} z\right) \in \mathcal{X}_{\leq}$. Continuing this process inductively, we obtain

$$
\left(\mathcal{T}^{n} x, \mathcal{T}^{n} z\right) \in \mathcal{X}_{\leq}
$$

Now, we proceed to show that

$$
\liminf _{n} p\left(\mathcal{T}^{n} z, \mathcal{T}^{n} x\right)=0
$$

Suppose $\liminf _{n} p\left(\mathcal{T}^{n} z, \mathcal{T}^{n} x\right)=\delta>0$. Since $\lim _{n \rightarrow \infty} p\left(\mathcal{T}^{n} z, \mathcal{T}^{n} z\right)=0$, then for arbitrary $\epsilon$ $(0<\epsilon<\delta)$, there exists $N_{1} \in \mathbb{N}$ such that for every $n>N_{1}$, we have $p\left(\mathcal{T}^{n} z, \mathcal{T}^{n} z\right)<\epsilon$. Also, since $\liminf _{n} p\left(\mathcal{T}^{n} z, \mathcal{T}^{n} x\right)=\delta>\epsilon>0$, then there exists $N_{2} \in \mathbb{N}$ such that for every $n>N_{2}$, we have $p\left(\mathcal{T}^{n} z, \mathcal{T}^{n} x\right)>\epsilon$. Therefore, for every $n>N=\max \left\{N_{1}, N_{2}\right\}$, we have

$$
\begin{aligned}
& \max \left\{p\left(\mathcal{T}^{n-1} z, \mathcal{T}^{n-1} x\right), \min \left\{p\left(\mathcal{T}^{n-1} z, \mathcal{T}^{n} z\right), p\left(\mathcal{T}^{n-1} x, \mathcal{T}^{n} x\right), p\left(\mathcal{T}^{n} z, \mathcal{T}^{n-1} z\right)\right.\right. \\
& \left.\left.p\left(\mathcal{T}^{n} x, \mathcal{T}^{n-1} x\right)\right\}\right\}=p\left(\mathcal{T}^{n-1} z, \mathcal{T}^{n-1} x\right) .
\end{aligned}
$$

Now, on using (b), for every $n>N$, we get

$$
\psi\left(p\left(\mathcal{T}^{n} z, \mathcal{T}^{n} x\right)\right) \leq \psi\left(p\left(\mathcal{T}^{n-1} z, \mathcal{T}^{n-1} x\right)\right)-\phi\left(p\left(\mathcal{T}^{n-1} z, \mathcal{T}^{n-1} x\right)\right) \leq \psi\left(p\left(\mathcal{T}^{n-1} z, \mathcal{T}^{n-1} x\right)\right) .
$$

Therefore, as $\psi$ is an altering distance function, we get the nonnegative sequence $\left\{p\left(\mathcal{T}^{n} z, \mathcal{T}^{n} x\right)\right\}$ is decreasing. As earlier, we can prove $\lim _{n \rightarrow \infty} p\left(\mathcal{T}^{n} z, \mathcal{T}^{n} x\right)=0$, which is indeed a contradiction to nonzeroness of $\delta$, implying thereby

$$
\liminf _{n} p\left(\mathcal{T}^{n} z, \mathcal{T}^{n} x\right)=0
$$

Also, since $\left(x^{*}, z\right) \in \mathcal{X}_{\leq}$, therefore using the arguments of the earlier case, we can prove

$$
\lim _{n \rightarrow \infty} p\left(\mathcal{T}^{n} z, \mathcal{T}^{n} x^{*}\right)=0
$$

and by lower semi-continuity $p\left(\mathcal{T}^{n} z, \cdot\right)$, we have

$$
\begin{aligned}
& p\left(\mathcal{T}^{n} z, \lim _{m \rightarrow \infty} \mathcal{T}^{m} x\right) \leq \liminf _{m \rightarrow \infty} p\left(\mathcal{T}^{n} z, \mathcal{T}^{m} x\right)=\alpha_{n} \quad \text { (say), } \\
& p\left(\mathcal{T}^{n} z, x^{*}\right) \leq \liminf _{m \rightarrow \infty} p\left(\mathcal{T}^{n} z, \mathcal{T}^{m} x^{*}\right)=\beta_{n} \quad \text { (say). }
\end{aligned}
$$


As $\lim _{n \rightarrow \infty} \alpha_{n}=\lim _{n \rightarrow \infty} \beta_{n}=0$, thus, in view of Lemma 1, we conclude that

$$
\lim _{n \rightarrow \infty} \mathcal{T}^{n}(x)=x^{*}
$$

This completes the proof.

Corollary 4 With the addition of condition (B) to the hypotheses of Corollary 1 (or Corollary 2, Corollary 3) the fixed point of $\mathcal{T}$ turns out to be unique. Moreover,

$$
\lim _{n \rightarrow \infty} \mathcal{T}^{n}(x)=x^{*}
$$

for every $x \in \mathcal{X}$ provided $x^{*} \in \mathcal{F}_{\mathcal{T}}$, i.e., the map $\mathcal{T}: \mathcal{X} \rightarrow \mathcal{X}$ is a Picard operator.

Corollary 5 With the addition of condition (B) to the hypotheses of Theorem 2, the fixed point of $\mathcal{T}$ turns out to be unique. Moreover,

$$
\lim _{n \rightarrow \infty} \mathcal{T}^{n}(x)=x^{*}
$$

for every $x \in \mathcal{X}$ provided $x^{*} \in \mathcal{F}_{\mathcal{T}}$, i.e., the map $\mathcal{T}: \mathcal{X} \rightarrow \mathcal{X}$ is a Picard operator.

\section{Illustrative examples}

In what follows, we furnish two illustrative examples wherein one demonstrates Theorem 1 on the existence of a fixed point, while the other one exhibits the uniqueness of the fixed point in respect of Theorem 3.

Example 2 Consider $\mathcal{X}=[0,1]$ equipped with the usual metric $d(x, y)=|x-y|$ for all $x, y \in \mathcal{X}$, and $p=d$ to be a $w$-distance on $(\mathcal{X}, d)$. Define an order relation $\preceq$ on $\mathcal{X}$ as

$$
x \preceq y \Leftrightarrow x=y \quad \text { or } \quad\left[x, y \in\{0\} \cup\left\{\frac{1}{n}: n=2,3, \ldots\right\} \text { with } x \leq y\right],
$$

where $\leq$ is usual order. Then it is clear that

$$
\mathcal{X}_{\leq}=\left\{(x, y) \in \mathcal{X} \times \mathcal{X}: x=y \text { or } x, y \in\{0\} \cup\left\{\frac{1}{n}: n=2,3, \ldots\right\}\right\} .
$$

Let $\mathcal{T}: \mathcal{X} \rightarrow \mathcal{X}$ be given by

$$
\mathcal{T}(x)= \begin{cases}0, & \text { if } x=0 \\ \frac{1}{n+1}, & \text { if } x=\frac{1}{n}, \\ \frac{\sqrt{2}}{2}, & \text { otherwise. }\end{cases}
$$

Obviously, $(\mathcal{X}, d, \preceq)$ is a complete partially ordered metric space. It is easy to see that $\mathcal{T}$ is nondecreasing. Also, there is $x_{0}=0$ in $\mathcal{X}$ such that $x_{0}=0 \preceq 0=\mathcal{T} x_{0}$, i.e., $\left(x_{0}, \mathcal{T} x_{0}\right) \in \mathcal{X}_{\leq}$, and $\mathcal{T}$ satisfies $\left(\mathrm{c}^{\prime}\right)$.

We now show that $\mathcal{T}$ satisfies (b) with $\psi, \phi:[0, \infty) \rightarrow[0, \infty)$ which are defined as

$$
\psi(t)=t^{2} \quad \text { and } \quad \phi(t)=t^{4} \quad(t \in[0, \infty))
$$


If $(x, y) \in \mathcal{X}_{\leq}$and $x=y$, then $p(\mathcal{T} x, \mathcal{T} y)=p(x, y)=0$. Otherwise, if $(x, y) \in \mathcal{X}_{\leq}$with $x \neq y$, then either $x=\frac{1}{n}, y=0$ or $x=\frac{1}{n}, y=\frac{1}{m}(m>n \geq 2)$, which evolve into two cases as follows.

Case 1. If $x=\frac{1}{n}(n \geq 2)$ and $y=0$, then

$$
\mathcal{M}_{\frac{1}{n}, 0}=\max \left\{p\left(\frac{1}{n}, 0\right), \min \left\{p\left(\frac{1}{n}, \mathcal{T} \frac{1}{n}\right), p(0,0), p\left(\mathcal{T} \frac{1}{n}, \frac{1}{n}\right), p(0,0)\right\}\right\}=\frac{1}{n}
$$

and

$$
\begin{aligned}
\psi\left(\mathcal{M}_{\frac{1}{n}, 0}\right)-\phi\left(\mathcal{M}_{\frac{1}{n}, 0}\right) & =\left(\frac{1}{n}\right)^{2}-\left(\frac{1}{n}\right)^{4}>\frac{1}{n^{2}}-\frac{1}{n^{2}(n+1)}=\frac{n}{n^{2}(n+1)} \\
& =\frac{1}{n(n+1)}>\frac{1}{(n+1)^{2}}=\psi(p(\mathcal{T} x, \mathcal{T} y)) .
\end{aligned}
$$

Case 2. Next, if $x=\frac{1}{n}$ and $y=\frac{1}{m}(m>n \geq 2)$, then

$$
\begin{aligned}
\mathcal{M}_{\frac{1}{n}, \frac{1}{m}} & =\max \left\{p\left(\frac{1}{n}, \frac{1}{m}\right), \min \left\{p\left(\frac{1}{n}, \mathcal{T} \frac{1}{n}\right), p\left(\frac{1}{m}, \mathcal{T} \frac{1}{m}\right), p\left(\mathcal{T} \frac{1}{n}, \frac{1}{n}\right), p\left(\mathcal{T} \frac{1}{m}, \frac{1}{m}\right)\right\}\right\} \\
& =\max \left\{\left|\frac{1}{n}-\frac{1}{m}\right|, \min \left\{\left|\frac{1}{n}-\frac{1}{n+1}\right|,\left|\frac{1}{m}-\frac{1}{m+1}\right|\right\}\right\} .
\end{aligned}
$$

For $m>n \geq 2$, we have

$$
\left|\frac{1}{n}-\frac{1}{n+1}\right| \leq\left|\frac{1}{n}-\frac{1}{m}\right|
$$

which is equivalent to

$$
\frac{1}{n(n+1)} \leq \frac{(m-n)}{m n}
$$

or

$$
\frac{m}{n+1} \leq(m-n)
$$

The preceding inequality holds as

$$
\frac{m}{n+1} \leq 1 \leq(m-n)
$$

so that

$$
\mathcal{M}_{\frac{1}{n}, \frac{1}{m}}=\left|\frac{1}{n}-\frac{1}{m}\right|
$$

Also (with $m>n \geq 2$ ),

$$
\psi(p(\mathcal{T} x, \mathcal{T} y)) \leq \psi\left(\mathcal{M}_{x, y}\right)-\phi\left(\mathcal{M}_{x, y}\right)
$$

or

$$
\left|\frac{1}{n+1}-\frac{1}{m+1}\right|^{2} \leq\left|\frac{1}{n}-\frac{1}{m}\right|^{2}-\left|\frac{1}{n}-\frac{1}{m}\right|^{4}
$$


or

$$
\frac{(m-n)^{2}}{(n+1)^{2}(m+1)^{2}} \leq \frac{(m-n)^{2}}{(m n)^{2}}-\frac{(m-n)^{4}}{(m n)^{4}}
$$

or

$$
\frac{(m-n)^{4}}{(m n)^{4}} \leq \frac{(m-n)^{2}}{(m n)^{2}}-\frac{(m-n)^{2}}{(n+1)^{2}(m+1)^{2}}
$$

or

$$
\frac{(m-n)^{2}}{(m n)^{2}} \leq \frac{((n+1)(m+1)-m n)((n+1)(m+1)+m n)}{(n+1)^{2}(m+1)^{2}} .
$$

Therefore,

$$
\left(\frac{1}{m}-\frac{1}{n}\right)^{2} \leq \frac{(n+m+1)((n+1)(m+1)+m n)}{(n+1)^{2}(m+1)^{2}} .
$$

Also, we can write

$$
\begin{aligned}
\left(\frac{1}{m}-\frac{1}{n}\right)^{2} & \leq \frac{1}{m^{2}} \leq \frac{1}{(n+1) m}=\frac{m}{(n+1) m^{2}} \leq \frac{m}{(n+1)(m+1)} \\
& \leq \frac{m}{(n+1)(m+1)}+\frac{1}{m+1}=\frac{m+n+1}{(n+1)(m+1)} \\
& \leq \frac{(m+n+1)(n+1)(m+1)}{(n+1)^{2}(m+1)^{2}}+\frac{(m+n+1)(m n)}{(n+1)^{2}(m+1)^{2}} \\
& =\frac{(n+m+1)((n+1)(m+1)+m n)}{(n+1)^{2}(m+1)^{2}},
\end{aligned}
$$

which amounts to say that the inequality (I) holds and so does the inequality (b) (of Theorem 1).

Thus, all the conditions of Theorem 1 are satisfied implying thereby the existence of a fixed point of the map $\mathcal{T}$ which are indeed two in number, namely 0 and $\frac{\sqrt{2}}{2}$. Here, it is worth pointing out that condition (B) does not hold in respect of this example.

We give another example that illustrates Theorem 3.

Example 3 Let $\mathcal{X}=\{0\} \cup\left\{\frac{1}{2^{n}}: n \geq 1\right\}$, where $(\mathcal{X}, d, \leq)$ is a complete partially ordered metric space with a metric $d$ and usual order $\leq$. Clearly, condition (B) holds in $\mathcal{X}$.

We define $p: \mathcal{X} \times \mathcal{X} \rightarrow[0, \infty)$ by $p(x, y)=y$. Let $\phi(t)=\frac{1}{4} t$ and $\psi(t)=\frac{1}{2} t$. Assume that $\mathcal{T}: \mathcal{X} \rightarrow \mathcal{X}$ by $\mathcal{T} x=\frac{x}{32}$ for any $x \in \mathcal{X}$. Obviously, $\phi$ and $\psi$ are altering distance functions, it is easy to see that $\mathcal{T}$ is nondecreasing and self-map. Also, there is $x_{0}=0$ in $\mathcal{X}$ such that $\left(x_{0}, \mathcal{T} x_{0}\right) \in \mathcal{X}_{\leq}$, and $\mathcal{T}$ satisfies $\left(\mathrm{c}^{\prime}\right)$ (of Theorem 3 ). Now, we show that $\mathcal{T}$ satisfies (b) (of Theorem 3). If $y=0$, clearly, condition (b) is satisfied. Now, suppose that $y=\frac{1}{2^{m}}$, then we have

$$
\begin{aligned}
\mathcal{M}_{x, \frac{1}{2^{m}}} & =\max \left\{p\left(x, \frac{1}{2^{m}}\right), \min \left\{p(x, \mathcal{T} x), p\left(\frac{1}{2^{m}}, \mathcal{T} \frac{1}{2^{m}}\right), p(\mathcal{T} x, x), p\left(\mathcal{T} \frac{1}{2^{m}}, \frac{1}{2^{m}}\right)\right\}\right\} \\
& =\frac{1}{2^{m}} .
\end{aligned}
$$


By making use of condition (b), one gets

$$
\psi p(\mathcal{T} x, \mathcal{T} y)=\psi\left(\frac{1}{32 \cdot 2^{m}}\right) \leq \psi\left(\frac{1}{2^{m}}\right)-\phi\left(\frac{1}{2^{m}}\right),
$$

so that

$$
\frac{1}{2}\left(\frac{1}{32 \cdot 2^{m}}\right) \leq \frac{1}{2}\left(\frac{1}{2^{m}}\right)-\frac{1}{4}\left(\frac{1}{2^{m}}\right)
$$

or

$$
\frac{1}{2^{m+6}} \leq \frac{1}{2^{m+1}}-\frac{1}{2^{m+2}}=\frac{1}{2^{m+2}} .
$$

The preceding inequality holds and so does the inequality (b) (of Theorem 3 ).

Thus, all the conditions of Theorem 3 are satisfied. We note that $x=0$ is a unique fixed point for $\mathcal{T}$. Moreover $\lim _{n \rightarrow \infty} \mathcal{T}^{n}(x)=\lim _{n \rightarrow \infty} \frac{x}{32^{n}}=0$.

\section{Competing interests}

The authors declare that they have no competing interests.

\section{Authors' contributions}

All authors contributed equally and significantly in this research work. All authors read and approved the final manuscript.

\section{Author details}

1'Department of Mathematics, Aligarh Muslim University, Aligarh, 202002, India. ${ }^{2}$ Permanent address: Mazandaran Province Education ORG, Mazandaran, Iran.

\section{Acknowledgements}

Both authors are grateful to two anonymous referees for their fruitful suggestions and observations.

Received: 9 July 2012 Accepted: 5 November 2012 Published: 7 December 2012

\section{References}

1. Khan, MS, Swaleh, M, Sessa, S: Fixed point theorems by altering distances between the points. Bull. Aust. Math. Soc. 30, 1-9 (1984)

2. Reich, S: Some fixed point problems. Atti Accad. Naz. Lincei, Rend. Cl. Sci. Fis. Mat. Nat. 57, 194-198 (1974)

3. Babu, GVR, Lalitha, B, Sandhya, ML: Common fixed point theorems involving two generalized altering distance functions in four variables. Proc. Jangieon Math. Soc. 10, 83-93 (2007)

4. Caristi, J: Fixed point theorems for mapping satisfying inwardness conditions. Trans. Am. Math. Soc. 215, 241-251 (1976)

5. Dutta, PN, Choudhury, BS: A generalization of contraction principle in metric spaces. Fixed Point Theory Appl. 2008 Article ID 406368 (2008)

6. Ekeland, I: Nonconvex minimization problem. Bull. Am. Math. Soc. 1, 443-474 (1979)

7. Kada, O, Suzuki, T, Takahashi, W: Nonconvex minimization theorems and fixed point theorems in complete metric spaces. Math. Jpn. 44, 381-391 (1996)

8. Nashine, HK, Altun, l: Fixed point theorems for generalized weakly contractive condition in ordered metric spaces. Fixed Point Theory Appl. 2011, Art. ID 132367 (2011)

9. Nieto, JJ, Rodríguez-López, R: Contractive mapping theorems in partially ordered sets and applications to ordinary differential equations. Order 22, 223-239 (2005)

10. Ran, ACM, Reurings, MCB: A fixed point theorem in partially ordered sets and some applications to matrix equations. Proc. Am. Math. Soc. 132, 1435-1443 (2004)

11. Rhoades, BE: Some theorems on weakly contractive maps. Nonlinear Anal. 47, 2683-2693 (2001)

12. Sastry, KPR, Babu, GVR: Some fixed point theorems by altering distances between the points. Indian J. Pure Appl. Math. 30, 641-647 (1999)

13. Alber, YI, Guerre-Delabriere, S: Principles of weakly contractive maps in Hilbert spaces. In: Gohberg, Y, Lyubich, Y (eds.) New Results in Operator Theory and Its Applications. Operator Theory, Advances and Applications, vol. 98, pp. 7-22. Birkhäuser, Basel (1997)

14. Takahashi, W: Existence theorems generalizing fixed point theorems for multivalued mappings. In: Thera, MA, Baillon, JB (eds.) Fixed Point Theory Appl. Pitman Research Notes in Mathematics Series, vol. 252, pp. 397-406. Wiley, New York (1991)

15. Altun, I, Simsek, H: Some fixed point theorems on ordered metric spaces and application. Fixed Point Theory Appl. 2010, Art. ID 621469 (2010) 
16. Suzuki, T: Generalized distance and existence theorems in complete metric spaces. J. Math. Anal. Appl. 253, 440-458 (2001)

17. Suzuki, T: Several fixed point theorems in complete metric space. Yokohama Math. J. 44, 61-72 (1997)

doi:10.1186/1687-1812-2012-222

Cite this article as: Imdad and Rouzkard: Fixed point theorems in ordered metric spaces via $w$-distances. Fixed Point Theory and Applications 2012 2012:222.

Submit your manuscript to a SpringerOpen ${ }^{\circ}$ journal and benefit from:

- Convenient online submission

- Rigorous peer review

- Immediate publication on acceptance

- Open access: articles freely available online

- High visibility within the field

- Retaining the copyright to your article

Submit your next manuscript at $>$ springeropen.com 